\title{
Colloque Les rythmes urbains (XVe-XIXe siècles) Appel à communication
}

\section{Olivier Zeller}

\section{(2) OpenEdition}

1 Journals

Édition électronique

URL : https://journals.openedition.org/ch/46

DOI : $10.4000 /$ ch.46

ISSN : 1777-5264

\section{Éditeur}

Comité historique du Centre-Est

\section{Édition imprimée}

Date de publication : 1 janvier 1998

ISSN : 0008-008X

\section{Référence électronique}

Olivier Zeller, "Colloque Les rythmes urbains (XVe-XIXe siècles) Appel à communication », Cahiers d'histoire [En ligne], 43-1 | 1998, mis en ligne le 12 mai 2009, consulté le 28 juin 2022. URL : http:// journals.openedition.org/ch/46 ; DOI : https://doi.org/10.4000/ch.46

Ce document a été généré automatiquement le 29 septembre 2020.

(c) Tous droits réservés 


\title{
Colloque Les rythmes urbains (XVe- XIXe siècles) Appel à communication
}

\author{
Olivier Zeller
}

1 Le Centre Pierre Léon d'histoire économique et sociale, UMR 5599 du CNRS organisera à Lyon, en avril 2000 (date à préciser), un colloque sur le thème des rythmes urbains.

2 Organisateur: Olivier Zeller, Professeur d'histoire moderne, Université LumièreLyon 2/Centre Pierre Léon.

3 Comité scientifique: MM. les Professeurs Yves Lequin, Denis Menjot, Daniel Roche, Jacques Rossiaud et Olivier Zeller.

\section{Thématique générale}

4 Si la problématique des rythmes a été largement sollicitée par l'histoire rurale, il n'en est pas de même en ce qui concerne l'histoire urbaine, généralement peu attentive aux rythmes spécifiques de la ville. Ceux-ci apparaissent surtout au sein d'études démographiques, sous les traits de l'inscription dans le temps de la natalité, de la nuptialité, de la mortalité et, plus rarement, de la morbidité. Les études économiques, quant à elles, peuvent être riches d'indications sur le calendrier des foires et des marchés. Classiquement, l'histoire de la criminalité est sensible aux lieux et aux heures. Enfin, la spécificité des pratiques et des symboles liés au jalonnement du temps a été décrite dans l' Histoire du dimanche des Français de Robert Beck, et ce n'est pas ironiser que d'espérer une histoire du lundi qui aurait beaucoup à dire sur les formes de sociabilité caractéristiques des villes à forte population ouvrière, la spécificité des autres jours de la semaine renvoyant moins à la vie urbaine dans sa globalité qu'aux rythmes propres des élites urbaines, fixant par exemple le calendrier des jours de réception. 
5 Se pencher sur les rythmes de la ville revient à poser une série de questionnements à la triple échelle du quotidien, de l'hebdomadaire et du saisonnier. Un premier inventaire, qui ne prétend pas à l'exhaustivité, peut en être dressé.

6 Saisir les rythmes urbains à l'échelle du quotidien suppose avant tout une connaissance de l'opposition entre jour et nuit. Dans la ville médiévale et moderne, la scansion en est formellement marquée par les horaires de fermeture et d'ouvertures des portes des remparts et par la prise ou la fin de service des unités du guet ou de la milice urbaine et, éventuellement, par la fermeture des quartiers par des chaînes ou des portails. Dans le même ordre de préoccupations se placent les ordonnances de police imposant un horaire de fermeture des portes des maisons. Cette distinction policière entre le temps du licite et de l'identificabilité et le temps de l'anonymat et de la suspicion revêt une portée toute particulière dès qu'il s'agit de l'appliquer à des espaces ou à des populations à statut spécial. Il convient donc de se pencher sur les rythmes propres d'alternance entre accessibilité et repli propres aux espaces retranchés qu'étaient les citadelles, les casernes et les ghettos, de même que sur les procédures d'exclusion nocturnes utilisées à l'encontre de groupes tolérés de jour, tels que les juifs à Strasbourg. Repérer des points de réglementation locale est ici un indispensable préliminaire. Ce dernier doit mener à une analyse des objets liés à la symbolique sécuritaire, du tir du canon à toutes les formes de sonneries de cloche ou, plus généralement, de signaux à destination collective plantant dans le paysage sonore urbain des jalons marquant la limite entre le licite et l'illicite. À cet égard, le cadre chronologique envisagé ne peut être compris qu'en termes d'évolution. Il s'agira donc de mesurer l'impact sur les réglementations de la victoire remportée sur les ténèbres par la lanterne urbaine, en attendant le bec de gaz. L'histoire des veilleurs, réveillematin et autres sereños, trop souvent annexée par le folklorisme ou le localisme, se doit d'être construite à une échelle européenne, puisque la démarche se doit de dépasser la constatation factuelle.

7 Il lui faudra donc aborder la question de la nuit urbaine sous l'angle des représentations : l'analyse des discours doit produire une meilleure connaissance des risques et des terreurs nocturnes, du phantasme de l'animal errant - les loups dans la ville - à l'éventualité de l'agression meurtrière par quelque bande se cachant le jour pour mieux méfaire la nuit; le XVIIe siècle est particulièrement riche de documents sur les « retraites " utilisées par les malfaiteurs (caves, carrières, catacombes et autres lieux souterrains). L'association entre malfaisance, activité nocturne et résidence souterraine est évidemment opposée à la valorisation morale du domicile protecteur et de la vie réglée au fil d'un temps carillonné. Tard dans le XVIIIe siècle, des connotations négatives resteront attachées aux déplacements infra-urbains nocturnes.

8 Il importera, en négatif, de définir la fonction et la signification des lieux et des pratiques de transgression nocturne : tavernes, bordels, ports, bas-quais, cimetières, fortifications à l'abandon et maisons de la périphérie, en attendant la zone. La transgression de l'ordre urbain peut prendre les formes d'une transgression de ses rythmes imposés et de la négation des impératifs attachés à ces rythmes : Réveillez-vous, gens qui dormez, priez Dieu pour les Trépassés, lançaient les réveille-matin lyonnais, réitérant sans cesse leur injonction d'associer la sérénité nocturne au rappel des fins dernières et participant de ce fait au découpage religieux du temps urbain. La nuit reste, dans ce domaine, le temps des cérémonies discrètes parce que n'existant qu'en 
vertu d'une tolérance: sépultures nocturnes de ceux de la R.P.R., remariages peu valorisés par l'Église.

Il est impossible, en tout état de cause, de faire l'économie d'une recherche précise, en horaire et en fréquence, de l'encadrement du temps urbain par les sonneries paroissiales et conventuelles, et de tenter d'objectiver l'ambiance "sonnante et carillonnante " évoquée par Pierre Goubert. Sans doute la lecture de mémoiresjournaux peut-elle permettre de se faire une idée du retentissement de ce rythme religieux sur la construction du rythme personnel d'existence. Mais il importe tout autant de suivre les progrès de la laïcisation du temps urbain. Collectivement, la mise en place des horloges municipales permet de prodiguer un service public davantage adapté à la vie économique que ne peuvent l'être les indications dispersées scandant la vie religieuse. Cette laïcisation passe également par l'appropriation personnelle d'instruments de mesure du temps, d'où l'intérêt d'une géographie horlogère tendant non seulement à situer l'exercice d'un métier rare dans le tissu urbain, en relation avec la répartition résidentielle des élites, mais également à le rechercher aux différents paliers de la hiérarchie urbaine. La Révolution finit par marquer le triomphe d'un temps déchristianisé et, de même que le calendrier républicain s'applique à effacer le référentiel mental grégorien, l'horloge citoyenne des édifices publics se substitue aux sons tombés des clochers. Les époques suivantes verront renaître cette concurrence, à ceci près que de nouveaux marqueurs du temps de la ville apparaîtront, à l'exemple des sirènes d'embauche des manufactures.

10 La connaissance des rythmes urbains du quotidien passe nécessairement par l'étude des activités économiques. Sur le plan normatif, les ordonnances abondent, qui fixent très précisément les heures d'ouverture et de fermeture des marchés, des grenettes, des tavernes et cabarets, quand ce n'est pas des boutiques. Mais une bonne restitution des activités du quotidien doit passer par une définition des temps propres à chaque métier : tripiers ambulants allant dès l'aube, journaliers et compagnons du bâtiment rassemblés au matin sur les lieux coutumiers de l'offre de travail, boulangers ouvrant boutique, femmes revenderesses exposant leurs légumes au retour de la campagne environnante, en fin de matinée. Au-delà du simple repérage dans le temps de la manifestation d'activités diverses, l'enjeu est ici de déterminer l'un des éléments de la culture urbaine. Il ne suffit pas, pour quiconque recherche une transaction matérielle ou de services, de connaître les lieux désignés par l'usage; le mode d'emploi des opportunités comporte nécessairement une maîtrise de l'heure qui rende possible l'échange souhaité.

11 Le rythme quotidien est aussi celui des activités ludiques. Les petits spectacles ont leur horaire, surtout quand leur activité est signalée par l'affiche. Dans le cas plus particulier des pratiques élitaires, il est net que les grandes villes de la seconde moitié du XVIIIe siècle voient s'affirmer un rythme quasi-quotidien réglé par l'horaire du théâtre, rythme de plus en plus dominateur puisque les grandes salles (Bordeaux, Lyon) des années 1780 finissent par ne plus connaître d'autre relâches que celle que les interdits religieux imposent. D'autres pratiques élitaires marquent le quotidien suivant des horaires définis : fréquentation du café, du billard, de la promenade publique. Une lecture des rythmes ludiques urbains peut se fonder sur l'exploitation des journaux d'annonces du XVIIIIe siècle.

12 Reste à prendre en considération la multiplication des infrastructures, essentiellement à compter du XVIIIe siècle. Comment les rythmes propres d'arrivée et de départ des 
coches de terre, de la grande poste, des turgotines, puis des premiers trains et des premiers transports en commun urbains se sont-ils surimposés aux rythmes préexistants, et comment les ont-ils modifiés?

13 Se pencher sur l'inscription dans la semaine des activités urbaines revient pour une bonne part à privilégier l'étude des caractères distinguant les jours ouvrables du dimanche et des jours régulièrement chômés (la saint Lundi). Plusieurs pistes, ici, sont ouvertes. Celle de l'étude des consommations alimentaires peut être imaginée à partir de livres de comptes de commerçants ou d'habitants : sources rares, à exhumer des fonds privés. Celle des rythmes cérémoniels est, en partie, plus aisée. Elle peut se fonder sur la statistique des mariages. Avec beaucoup de précautions, elle peut prendre en compte le rythme des baptêmes chaque fois qu'ils ne suivent pas systématiquement la naissance puisque la pratique de l'ondoiement de commodité permet une libre fixation de la date de célébration des "cérémonies complémentaires ». Le cas des sépultures est plus délicat, le rythme des enterrements étant très fortement conditionné par celui des décès. Il n'est possible d'utiliser la notion de rythme qu'à partir du moment où les conditions matérielles de conservation de corps se mettent à offrir une certaine latitude de fixation des obsèques. D'une manière générale, l'appartenance religieuse induit un rythme cultuel spécifique. Du temps catholique urbain se démarque un temps réformé urbain. D'une part, les injonctions religieuses diffèrent. D'autre part, la ségrégation spatiale des cultes livre le dimanche urbain aux catholiques tandis que les réformés doivent quitter la ville pour gagner un temple rejeté en banlieue, à l'exemple de Charenton. Lorsqu'une division formelle de l'espace partage l'espace urbain entre quartiers chrétiens et quartiers juifs, à l'instar d'Avignon et des villes du Comtat, deux rythmes hebdomadaires s'opposent : celui du dimanche, et celui du sabbat. L'une des formes de persécution infligées par l'Église consiste justement à imposer aux juifs une assistance dominicale à de véhémentes prédications, brisant de force leur rythme propre par irruption d'un rythme exogène. Mais il importe de repérer, à nouveau, les effets de la déchristianisation. Si la position dans la semaine de beaucoup des fêtes "corporatives » organisées par les confréries de métier ou les communautés de métier dépendait du hasard de la situation dans le calendrier de la fête du saint éponyme, qu'en fut-il de toutes les manifestations professionnelles ultérieures, ou simplement différentes? La problématique du temps se complète nécessairement d'une problématique de l'espace. Relisons Ménétra. La narration de ses séjours lyonnais donne un bel exemple d'investissement dominical d'espaces festifs spécifiques tels que l'île Barbe; et l'on connaît par ailleurs l'habitude qu'avaient les ouvriers en soie lyonnais de sortir de Lyon pour se rendre aux guinguettes des Charpennes, un espace ludique qui se muait en espace de transgression lors des grèves organisées, comme en 1786. La quantification de ces flux dominicaux est imaginable. De même que l'on connaît les rythmes de fréquentation des théâtres londoniens du XVIe siècle par le truchement des comptes des passeurs sur la Tamise, il suffit de mettre en œuvre les comptes d'un péager (ceux d'un pont, par exemple) pour mesurer les déplacements dominicaux.

14 L'étude des autres rythmes festifs doit accorder une large place au temps théâtral ${ }^{1}$. Il ne suffit pas ici de repérer l'intensification de l'offre de spectacle au cours du XVIIIe siècle qui amène à ouvrir pratiquement chaque jour de la semaine; il importe également d'établir la sociologie du public et de la mettre en double relation avec le nombre de billets de porte vendus chaque jour et avec la stratégie de composition du répertoire. Le vendredi est-il partout un jour de tragédie plutôt creux - sauf quand la 
pièce est de Voltaire - et le dimanche est-il partout le jour du théâtre lyrique le plus léger, destiné à un public socialement élargi ?

D'autres formes majeures de sociabilité structurent le temps hebdomadaire de la cité. Les jours de tenue des ateliers maçonniques - voir les tableaux imprimés des loges anglaises - les jours de réunion des membres des cercles et des sociétés savantes sont autant d'éléments de première importance pour la connaissance des rythmes propres aux élites.

Ceux-ci, pour une large part, dépendent de l'activité d'un certain nombre d'institutions. Municipalités, chambres de commerce, bureaux de charité, rectorats d'hôpitaux, juridictions de toute espèce ont leur calendrier propre. C'est dire l'intérêt d'un dépouillement systématique de séries d'almanachs dans la perspective d'une recherche de la spécificité d'occupation tacitement assignée à chaque jour ou groupe de jours dans la semaine et intériorisée par la culture élitaire urbaine.

Plus discrets semblent les rythmes hebdomadaires populaires. Classiquement, l'étude des sources policières et judiciaires semble s'imposer : une statistique des délits et des crimes en fonction des lieux, des jours et des activités est réalisable à coup sûr. Mais de nombreuses expériences restent à tenter dans des champs très différents. Existe-t-il une répartition significative suivant les jours de la semaine des abandons d'enfant, des entrées à l'hôpital, des distributions aux indigents ? Comment les activités propres aux milices urbaines - manœuvres, tours de garde - s'insèrent-elles dans la semaine ? Faute de pouvoir longtemps identifier les suicides en tant que tels, trouve-t-on des régularités dans la temporalité des noyades?

18 Il reste enfin à baliser l'hebdomadaire urbain dans la banalité de la vie ordinaire, de la répartition par le locataire principal des jours de lessive entre les habitants de son immeuble jusqu'aux jours d'ouverture des marchés, des boucheries, des étuves, des bains, sans oublier l'impact des activités non quotidiennes, à l'exemple des passages de coches d'eau. Le calendrier hebdomadaire peut également distinguer des jours de protectionnisme économique, réservant le droit de vendre aux marchands de la ville, et des jours de relative ouverture permettant aux marchands forains (en particulier aux boulangers) d'assurer à la ville un approvisionnement d'appoint sous un régime de concurrence étroitement contrôlée.

19 Les rythmes annuels se placent à un niveau tout différent. Ils ne concernent pas obligatoirement une même population. L'effet de spinning door des flux migratoires vers et hors de la ville en fait un lieu de passage continuel où les implantations sont souvent éphémères, de l'ordre de quelques semaines à celui de quelques mois. Suivant les trajectoires individuelles, le vécu de l'urbain se trouve segmenté au fil du calendrier migratoire. Tel ne connaîtra la ville que durant la belle saison, à l'exemple de beaucoup de migrants du bâtiment. Tel autre, inversement, ne l'habitera que l'automne et l'hiver; il peut s'agir de quelque membre d'une maison aristocratique partageant l'année entre son hôtel urbain et le séjour sur ses terres ${ }^{2}$. Il n'est sans doute pas exagéré d'avancer que l'appropriation des rythmes annuels spécifiques d'une ville est un gage d'intégration culturelle. La contre-preuve en est administrée par le regard des voyageurs qui, placés par définition hors des rythmes longs, ne peuvent voir la ville que dans l'instantanéité.

20 À nouveau, la problématique suscite un inventaire à la Prévert. Il est des rythmes formels, institutionnalisés, hyper-signifiés. Le temps du religieux, en premier lieu, est marqué par les succession des fêtes, des processions, des temps d'interdictions. 
Secondairement, il se traduit en termes de consommation : le Carême, en particulier, voit passer les baux de la «ferme de la chair » conférant le monopole de la vente de viande à un boucher. La profession connaissant, de ce fait, une année amputée de 45 jours, elle module ses activités en fonction de ce rythme, passant par exemple des contrats avec les tanneurs valables de Pâques au Carême. Le temps religieux est censé se traduire par une chute des conceptions durant l'Avent et le Carême: les études démographiques soulignent quasiment toutes le faible impact de l'abstinence de décembre et l'affaiblissement de l'observation du Carême. Une synthèse reste à dresser.

Le temps politique est également un temps sonné et trompeté. Il dépend du mode de fonctionnement des institutions urbaines, et chaque ville et un cas d'espèce. Assemblées générales, assemblées de notables, élections, ou simulacres d'élections, proclamations, messes solennelles de requiem, revues de la milice, visites en corps aux collège, aux ordres religieux, aux hôpitaux, aux prisons, pèlerinages, ouvertures de foire se répétant d'année en année suivant un rite quasiment immuable. Il reste que chaque cérémoniel impose des échéances qui ne correspondent pas seulement aux fonctions de représentation, mais qui découlent du rythme de renouvellement des personnels.

Le temps judiciaire est sans nul doute un temps majeur de la vie urbaine. Ne laissant sur place que les officiers des vacations chargés d'expédier les affaires courantes, les magistrats désertent la ville durant les vacances, d'où un ralentissement connu, mais peu mesuré, de nombre d'activités urbaines. La clientèle des élites judiciaires se met à manquer aux métiers de luxe, les plaideurs cessent d'enrichir logeurs et aubergistes. Avocats et procureurs ne sont plus sollicités que par un moindre nombre de plaideurs; les théâtres se vident, et abaissent leurs tarifs. Simultanément, une grande part des élites déserte la ville avec d'autant moins d'inconvénients que les procès sommeillent un vigilant procureur gardant des «surprises » et des «ordonnances de défaut »- et que les réseaux ordinaires de relations se trouvent momentanément désactivés. Il se dégage donc un temps des sociabilités doté de rythmes propres, les relations de la campagne pouvant briser avec le cadre des sociabilités urbaines ordinaires, et ces voisinages saisonniers s'appuyant sur des pratiques ludiques spécifiques que révèlent nombre d'agencements d'intérieur ou de jardin.

Le temps économique se décrit en termes de fluctuations répétées. C'est un lourd truisme que de répéter combien les villes dépendent du cours d'eau qui les longe ou les traverse. Par là s'exerce la contingence. Les transports d'hommes et de biens cessent lors des crues, des étiages et des prises des eaux par les glaces, de même que les activités industrielles fonctionnant grâce à « l'artifice » de machines mues par les aubes de moulins. L'approvisionnement frumentaire lui-même peut se trouver compromis par l'arrêt des moulins à farine, au point que les moutures d'automne et le contrôle des stocks de farine à l'approche d'octobre constitue des préoccupations édilitaires majeures.

Le rythme annuel des consommations dépend également des conditions matérielles de transport et de conservation des comestibles. Les glacières n'offrent qu'un palliatif limité aux effets des chaleurs, et, en particulier, la consommation urbaine de poisson est soumise à des rythmes saisonniers: les chasse-marées sont périodiquement contraints de cesser leur activité, et les réglementations municipales édictent généralement des prohibitions estivales. À partir du XVIIIe siècle, période de multiplication des brasseries, il est un temps de la bière et il est un temps du vin. Mais 
l'on sait que les consommations carnées fluctuent également au long de l'année, hors Carême : il est un temps du porc, un temps du veau, un temps du mouton ${ }^{3}$

D'une manière très générale, les relations matérielles entre la ville et ses différents horizons sont soumises aux conditions de transport par voie de terre comme par voie d'eau. Le risque des gués, la boue des chemins et les intempéries conditionnent à l'évidence les flux commerciaux : par exemple, les foires de Lyon de Pâques et d'août sont connues pour avoir été largement mieux achalandées que celles de Toussaint et des Rois. Tout, ici, peur être modifié par les conditions géographiques. Négativement, les villes en site de piémont peuvent se muer en culs-de-sac lors de la fermeture des cols. Positivement, les villes maritimes et, plus particulièrement, les villes d'estuaire restent plus régulièrement en relation avec de vastes espaces.

La production peut se trouver soumise aux rythmes caractéristiques de ces différents flux. Les espaces spécialisés de déchargement et de stockage que sont les ports et les entrepôts peuvent jouer un rôle de régulation des flux de matières premières; mais un autre déterminant essentiel est constitué par l'état du marché du travail local. Durant toute la modernité, et tard dans l'époque contemporaine, les trajectoires individuelles sont souvent marquées par une forte mobilité correspondant à l'exercice d'emplois saisonniers ou d'activités pratiquées durant quelque mois, ceci en alternance avec des phases de retour au pays. De tels phénomènes s'observent par exemple à l'égard des Savoyards, frotteurs de parquet, ramoneurs ou marchands ou à celui des Limousins scieurs, maçons ou tailleurs de pierre. Le bâtiment, secteur primordial, emploie ainsi une main d'œuvre en renouvellement quasi permanent. Mais la pratique concerne aussi les serviteurs, dont le turn-over est extrêmement fort, au point que les niveaux des gages puissent connaitre des fluctuations conjoncturelles. Mobilité, également de la main d'œuvre agricole. Si les ruraux viennent travailler en ville, les urbains peuvent également gagner le plat pays pour aider aux moissons ou aux vendanges. Tout dépend, ici, de la position de la ville. En temps ordinaire, une part importante de la population de beaucoup de petites villes est spécialisée dans des activités agricoles, et l'époque des grandes façons culturales voit se réaliser un gonflement temporaire de ces effectifs par concentration du potentiel flottant de main d'œuvre que représentent, entre autres, les journaliers, les non-qualifiés, peut-être les veuves et les enfants. Le degré d'implication d'une ville dans son terroir ne se mesure donc pas seulement à l'aune de l'importance moyenne de son "secteur primaire ", telle que peuvent l'établir des comptages dans les recensements ou les rôles fiscaux. Elle s'exprime également par l'écho que trouvent les rythmes ruraux dans ses propres temporalités.

Autant dire que, pour une large part, les rythmes urbains sont des rythmes démographiques. La démographie «classique » a nettement montré les mouvements du nombre des baptêmes, des mariages et des sépultures, mouvements qui ne sont pas nécessairement traductibles en taux dès que la mobilité géographique est accentuée. Des chantiers importants restent néanmoins ouverts. Au premier rang se place l'étude des morbidités et des mortalités saisonnières, elles-mêmes liées au contexte écologique urbain : la typhoïde, par exemple, résulte de la conjonction de conditions climatiques et hydrologiques. Par ailleurs, la mobilité géographique, perçue en négatif grâce aux échecs des tentatives de reconstitution paroissiale, est fortement liée aux pratiques d'habitat et, tout particulièrement, aux rythmes scandés par les échéances des baux locatifs. Surtout, une large part doit être accordée à l'étude des flux humains en provenance ou à destination de l'extérieur de la ville. Une connaissance exhaustive des 
mouvements aux portes de la cité reste du domaine de l'onirisme, même s'il n'est pas interdit de penser que, très exceptionnellement, des sources directes aient pu exister ça et là, ne fût-ce qu'en raison de circonstances particulières. Faute de mieux, la démarche essentielle renvoie donc à la problématique de l'hospitalité urbaine. Celle-ci se parcourt en reconstituant les flux d'admission dans les hôpitaux, en repérant les mobilités scolaires, les déplacements de groupes de pèlerins, les séjours de militaires en quartier d'hiver - le passage des troupes dans les villes d'étape scandant, pour elles, un rythme essentiel. Enfin, les migrations saisonnières élitaires, au fort poids économique et symbolique, méritent une approche quantitative.

Qu'il s'agisse des rythmes quotidiens, hebdomadaires ou annuels, la synthèse parait lointaine, tant sont nombreux les questionnements à mettre en œuvre. Il parait indispensable, en première phase, de se livrer à la réalisation de monographies de repérage destinées à établir une meilleure connaissance des rythmes économiques, migratoires, festifs et autres. L'hypothèse de départ suppose l'existence de convergences, de régularités; l'éventuelle mise en évidence de villes arythmiques fournirait d'utiles contre-exemples, pour peu qu'elles puissent être rapprochées d'évidentes spécificités locales. Une double tentative de mise en relation pourra alors avoir lieu: d'une part, entre les différents rythmes d'une même ville et, d'autre part, entre les types repérés de rapport de l'urbain au temps et les tailles et les fonctions des villes constituant l'échantillon.

En deuxième phase, une rafale de questionnements pourra s'appliquer à l'évolution de ces rythmes dans le long terme. Trois événements paraissent susceptibles d'avoir engendré de profonds bouleversements des rythmes: la Réforme, la Révolution industrielle, et, dans une moindre mesure, la Révolution française. Dans le temps long, comment l'amélioration de l'éclairage privé et public a-t-elle mené à l'annexion de la nuit par le temps « utile» de l'économie ? Comment la révolution des transports a-telle levé les hypothèques liées à l'emploi de la voie d'eau ? Dans quelle mesure les villes qui donnaient écho aux rythmes de la campagne se sont-elles affranchies de leur influence? In fine, l'évolution à long terme s'est-elle traduite par une banalisation du temps urbain au fil de rythmes de plus en plus indifférents aux saisons, aux heures et aux jours?

Le colloque s'attachera à repérer toutes les pratiques urbaines s'inscrivant régulièrement dans un rythme quotidien, hebdomadaire ou annuel. Ceci amènera à considérer par exemple :

- la réglementation du temps urbain

- les marqueurs sonores du quotidien

- les rythmes ludiques du quotidien (spectacles, promenades, etc.)

- les rythmes religieux et festifs dans la semaine

- les rythmes hebdomadaires institutionnels (municipalités, administrations hospitalières,

etc.)

- les rythmes plus spécifiquement populaires (tours de milice, marchés, règlements

d'immeuble, etc.)

- les rythmes annuels :

- modulation des flux migratoires

- chronologie des abandons d'enfants, de la criminalité

- rythmes de production et de consommation

- rythmes religieux 
- temps des élites (vacances judiciaires, migrations ville-campagne, calendrier des sociétés savantes, déroulement de l'année théâtrale, etc.)

- rythmes démographiques

- les représentations des rythmes urbains (d'après des mémoires-journaux, par exemple).

31 Contacts :

Olivier ZELLER, Centre Pierre Léon

MRASH, 14 avenue Berthelot, 69363 Lyon cedex 07, France

tél. 0472726427 Fax : 0472726424

\section{NOTES}

1. Voir, par exemple : Olivier ZELLER, «L'intensification de la vie théâtrale à Lyon (1761-1788) », dans Cahiers d'histoire, tome 42, 1997, n², pp. 193-216.

2. Voir, par exemple: François-Joseph RUGGIU, Les élites et les villes moyennes en France et en Angleterre (XVIIe-XVIIIe siècles), Paris, L'Harmattan, 1997, 356 p.

3. Bernard GARNIER, "Les marchés aux bestiaux : Paris et sa banlieue », dans Éric BARATAY et Jean-Luc MAYAUD [dir.], L'animal domestique, XVIe-XXe siècles.- Cahiers d'histoire, tome $42, \mathrm{n}^{\circ} 3-4$, 1997, pp. 575-612.

\section{AUTEUR}

\section{OLIVIER ZELLER}

Centre Pierre Léon-Université Lyon 2 